\title{
Determination of the absorption constant in the interband region by photocurrent measurements
}

\author{
Journal Article \\ Author(s): \\ Mosimann, R.; Haertle, D.; Jazbinsek, Mojca; Montemezzani, G.; Günter, Peter \\ Publication date: \\ 2006 \\ Permanent link: \\ https://doi.org/10.3929/ethz-b-000001052 \\ Rights / license: \\ In Copyright - Non-Commercial Use Permitted \\ Originally published in: \\ Applied Physics B 83(1), https://doi.org/10.1007/s00340-006-2134-0
}




\author{
R. MOSIMANN \\ D. HAERTLE \\ M. JAZBINSEK \\ G. MONTEMEZZANI* \\ P. GÜNTER
}

\title{
Determination of the absorption constant in the interband region by photocurrent measurements
}

\author{
Nonlinear Optics Laboratory, Swiss Federal Institute of Technology, 8093 Zurich, Switzerland
}

\begin{abstract}
Received: 13 October 2005/
Revised version: 22 December 2005

Published online: 3 February 2006 • C) Springer-Verlag 2006

ABSTRACT We determined high absorption constants of crystals from photocurrent measurements within the interband absorption region $\left(10-10^{4} \mathrm{~cm}^{-1}\right)$. The method has been demonstrated in the interband absorption regime near $530 \mathrm{~nm}$ in $\mathrm{Sn}_{2} \mathrm{P}_{2} \mathrm{~S}_{6}$, a novel infrared sensitive photorefractive material, and in the interband absorption regime near $257 \mathrm{~nm}$ of near stoichiometric $\mathrm{LiTaO}_{3}$. Besides the verification of older measurements with our new technique, precise absorption data for $\mathrm{Sn}_{2} \mathrm{P}_{2} \mathrm{~S}_{6}$ in the wavelength range $488-514 \mathrm{~nm}$ are presented.
\end{abstract}

PACS 42.70.Nq; 72.41.+w; 78.20.Ci

\section{$1 \quad$ Introduction}

Light emitting diodes, photodetectors, electroabsorption modulators [1], solar cells and many other photonic devices involve transitions of charges between bands, and thus work in a region of high light absorption. Also holographic applications based on the interband photorefractive effect such as multiple quantum well devices [2, 3], incoherent-to-coherent optical converters [4], light-induced waveguides [5], high-frame-rate joint Fourier-transform correlators [6] and dynamically reconfigurable wavelength filters [7], operate beyond the absorption edge with absorption constants up to $10^{3} \mathrm{~cm}^{-1}$. In this region, the absorption constant is not easily measured, but is nevertheless of crucial importance for the underlying basic physical mechanisms and the applications.

The most common technique for measuring absorption constants in the order of $10-10^{4} \mathrm{~cm}^{-1}$ is a direct measurement of the transmission of a thin sample. This method is quite precise but often requires a thin plate of only a few $\mu \mathrm{m}$ thickness.

If the light at the wavelength of interest induces a secondary physical effect, it is possible to determine the absorption constant indirectly by a scanning method. For example,

Fax: +41-44-6331056, E-mail: roger.mosimann@phys.ethz.ch

*Present address: Laboratoire Matériaux Optiques, Photonique et Systèmes (LMOPS, UMR CNRS 7132), University of Metz and Supélec, 57070 Metz, France if the secondary effect is a light-induced absorption at another wavelength, we can determine the absorption constant by depth scanning of the transmitted intensity of the light at the second wavelength from the side of the crystal, while illuminating the crystal from the top with the wavelength of interest $[8,9]$. A similar method is based on scanning the diffraction efficiency of holographic gratings at different depths instead of using the induced absorption [10]. The basic principle of the scanning method is applicable for the absorption measurement if the strength of the secondary physical effect is a monotonic function of the light intensity. These techniques can reach a precision of about 25 percent, but very sharp polished edges of the crystal are needed, since the measurement involves probing light traveling only a few micrometers below the crystal surface. If the secondary effect is light-induced absorption, we refer to this technique here as the induced absorption method.

In ferroelectrics, the absorption constant can also be determined by measuring the transient pyroelectric current due to changes in the ferroelectric polarization produced by heating the sample through the absorption of light [11]. This method, however, usually requires very precise electrometers, the prior knowledge of several material constants, and is very sensitive to external influences, so that it has to be done in a vacuum chamber.

A different approach for determining the absorption constant is based on the reflectivity measurements. Either one measures the wavelength dependence of the reflectivity at normal incidence and then evaluates the absorption using the Kramers-Kronig relation, or one measures the angular dependence of the reflectivity at a certain wavelength and evaluates the absorption constant using the Fresnel formulas [12,13]. Both of these methods are only well suited for very high absorption constants in the order of $10^{4}-10^{5} \mathrm{~cm}^{-1}$ and they usually show limited accuracy.

In semiconductors, the photoconductivity measurement is a standard method to determine the absorption edge and the absorption constants in the transparent region [14]. Here we determine the absorption constant beyond the absorption edge by measuring the photocurrent in bulk crystals. Compared to the existing methods, it does not require any special crystal preparation or the knowledge of other material constants.

We first present in Sect. 2 a theoretical background of the proposed method. In Sect. 3 we report the measurements 
of the photocurrent for $\mathrm{Sn}_{2} \mathrm{P}_{2} \mathrm{~S}_{6}$ and the determination of the absorption constant at the wavelengths of $\lambda=514 \mathrm{~nm}$, $\lambda=501 \mathrm{~nm}, \lambda=496 \mathrm{~nm}$ and $\lambda=488 \mathrm{~nm}$ and compare the obtained values to the results of a direct transmission measurement using a thin plate of $45 \mu \mathrm{m}$ thickness. The absorption coefficients were also determined by this photocurrent method for near-stoichiometric $\mathrm{LiTaO}_{3}$ at $\lambda=257 \mathrm{~nm}$ and the results were compared to published data.

\section{Theoretical}

We consider a photoconducting dielectric crystal illuminated by light with photon energy larger than the band gap of the material. We are interested in the photocurrent generated by bandgap light with an applied electric field $E$ and on how this photocurrent depends on the absorption constant $\alpha$ if one of the sample surfaces is homogeneously illuminated. Figure 1a shows the orientation of the crystal and the coordinate system we use for our calculations and experiments with $\mathrm{Sn}_{2} \mathrm{P}_{2} \mathrm{~S}_{6}$ and Fig. $1 \mathrm{~b}$ shows the same for the experiments with $\mathrm{LiTaO}_{3}$. In the case of $\mathrm{Sn}_{2} \mathrm{P}_{2} \mathrm{~S}_{6}$ the light is incident on the surface normal to the $z$ axis and the field is applied parallel to the $x$ axis of the crystal. For $\mathrm{LiTaO}_{3}$ the light is incident on the surface normal to the $x$ axis and the field is applied parallel to the $z$ axis of the crystal.

According to the theoretical analysis of charge transport induced by interband illumination in photoconductive crystals [10] we can distinguish two different regimes, which are identified by a specific characteristic exponent $\kappa$ for the increase of the photoconductivity $\sigma$ with light intensity $I$ $\left(\sigma \propto I^{\kappa}\right)$. For low light intensities $I$ and considering only one deep impurity level (due to intrinsic defects or dopants) inside the band gap of the material, carriers recombine predominantly into the impurity level. The recombination rate does not depend on light intensity, which leads to a linear increase of the photoconductivity with light intensity, similarly to conventional off-resonant charge excitation [15]. For large light intensities $I$ the interband regime becomes dominant, as was also confirmed experimentally in $\mathrm{KNbO}_{3}$ [10] and $\mathrm{LiTaO}_{3}$ [9]. In this regime charge carriers recombine from the valence to the conduction band and the recombination rate increases with intensity. As a result the steady-state freehole concentration $p_{0}$ and the free-electron concentration $n_{0}$ follow the same square root intensity dependence $n_{0} \cong p_{0} \cong$ $\sqrt{g I / \gamma_{\text {dir }}}[10]$, where $\gamma_{\text {dir }}$ is the band-to-band recombination rate and $g$ the photoexcitation constant. The photoexcitation is

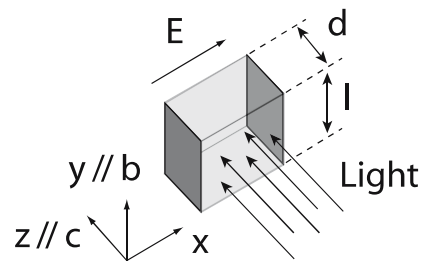

a

FIGURE 1 Coordinate system used and orientation of the crystallographic axes $a, b, c$. (a) shows the coordinate system and orientation of the crystal axes for $\mathrm{Sn}_{2} \mathrm{P}_{2} \mathrm{~S}_{6}$, where the $x$ axis is shifted by $15^{\circ}$ with respect to the crystallographic $a$ axis. (b) shows the coordinate system and the orientation of the crystallographic axes for $\mathrm{LiTaO}_{3}$ proportional to the absorption constant $\alpha$ as $g=\alpha / h \nu$, where $h$ is the Planck constant, $v$ the frequency and $\alpha$ the absorption constant. The photoconductivity $\sigma(z)$ is then given by:

$\sigma(z)=e\left(p_{0} \mu_{\mathrm{h}}+n_{0} \mu_{\mathrm{e}}\right) \propto e\left(\mu_{\mathrm{h}}+\mu_{\mathrm{e}}\right) \sqrt{\frac{\alpha I(z)}{v}} \propto \sqrt{\frac{\alpha}{v}} \mathrm{e}^{-\frac{\alpha z}{2}}$,

where $e$ is the electron charge, $\mu_{\mathrm{e}}$ the mobility of the electrons and $\mu_{\mathrm{h}}$ the mobility of the holes and where we assumed an exponential decrease of intensity in the beam propagation direction $z$ as $I(z)=I_{0} \mathrm{e}^{-\alpha z}$. The spatially integrated electric current $J$ measured by an amperemeter in series with the crystal can now be calculated as:

$J=\int_{0}^{d} j(z) l \mathrm{~d} z=$ const $E \sqrt{\frac{\alpha}{v}} l \int_{0}^{d} \mathrm{e}^{-\frac{\alpha z}{2}} \mathrm{~d} z$

where $l$ is the length of the crystal in the $y$ direction and $d$ the thickness in the $z$ direction, $j(z)=\sigma(z) E$ is the depthdependent electric current density for a field applied parallel to the $x$ direction and const is a proportionality factor including intrinsic material constants. By solving the last integral and considering $d \gg 1 / \alpha$, we get

$J \propto \frac{1}{\sqrt{\alpha \nu}}$

which shows that the measured current is inversely proportional to the square root of the absorption constant $\alpha$. This relationship is valid if the wavelength and intensity are such that the interband processes dominate, which means that the photoconductivity increases according to the square root of the intensity. In this case (3) can be used to determine the absorption constant by a comparative process provided that one absorption constant (at another wavelength or for a different polarization) has been determined directly by another method. The basic principle is schematically shown in Fig. 2 for the case, where the absorption constant $\alpha_{y}$ is smaller than $\alpha_{x}$, and where $\alpha_{y}^{\lambda_{1}}$ can be measured directly. Its wavelength $\left(\lambda_{1}\right)$ is typically chosen close to the transparency range, where the absorption can be determined by standard methods with high

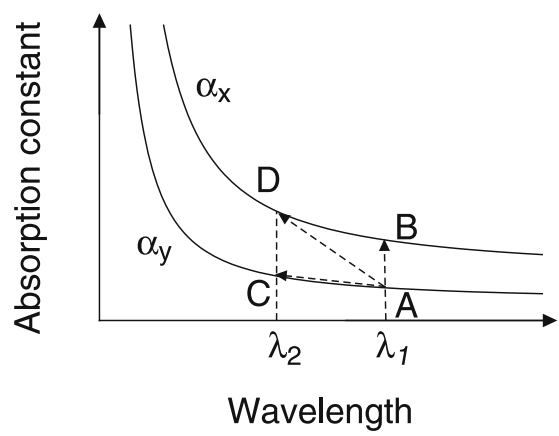

FIGURE 2 A schematic absorption spectra showing the principle of our method. For point A the lower absorption constant $\alpha_{y}^{\lambda_{1}}$ can be determined by direct transmission measurements. By using (4)-(6) one can calculate the large absorption constants $\alpha_{x}^{\lambda_{1}}, \alpha_{y}^{\lambda_{2}}, \alpha_{x}^{\lambda_{2}}$ etc. (points B-D) by measuring the photocurrents at the corresponding wavelengths and polarizations 
precision. Then the photocurrents $J_{x}^{\lambda_{1}}, J_{x}^{\lambda_{2}}, J_{y}^{\lambda_{1}}$ and $J_{y}^{\lambda_{2}}$ for the same intensity $\left(J \propto \sqrt{I_{0}}\right)$, for both wavelengths $\lambda_{1}$ and $\lambda_{2}$ and both polarizations ( $x$ and $y$ ) are measured. By using the following relationships, derived from (3),

$$
\begin{aligned}
& \sqrt{\frac{\alpha_{y}^{\lambda_{1}}}{\alpha_{x}^{\lambda_{1}}}}=\frac{J_{x}^{\lambda_{1}}}{J_{y}^{\lambda_{1}}}, \\
& \sqrt{\frac{\alpha_{y}^{\lambda_{1}}}{\alpha_{x}^{\lambda_{2}}}} \sqrt{\frac{\nu_{1}}{v_{2}}}=\frac{J_{x}^{\lambda_{2}}}{J_{y}^{\lambda_{1}}}, \\
& \sqrt{\frac{\alpha_{y}^{\lambda_{1}}}{\alpha_{y}^{\lambda_{2}}}} \sqrt{\frac{\nu_{1}}{\nu_{2}}}=\frac{J_{y}^{\lambda_{2}}}{J_{y}^{\lambda_{1}}}
\end{aligned}
$$

one can determine the absorption constants $\alpha_{x}^{\lambda_{1}}, \alpha_{y}^{\lambda_{2}}$ and $\alpha_{x}^{\lambda_{2}}$. In many cases these absorption constants are much too large to be determined directly by the classical methods as $\alpha_{y}^{\lambda_{1}}$ in our example. Note that the factor $\sqrt{\nu_{1} / \nu_{2}}$ in (5) and (6) takes into account that light of the same intensity $I_{0}$ does not contain the same number of photons at different wavelengths. If the reflection losses differ considerably at different wavelengths or polarizations because of different refractive indices, one should consider it when normalizing the current to the input intensity $\left(J \propto \sqrt{I_{0}}\right)$ considering $I_{0}=(1-R) I_{\text {ext }}$, where $R=(n-1)^{2} /(n+1)^{2}$ is the reflectivity, $n$ the refractive index and $I_{\text {ext }}$ the external light intensity.

\section{Experimental verification}

We verified the proposed method for two different materials, where band-to-band photorefraction [10] is a very efficient recording process. The first material we used was the narrow-bandgap ferroelectric crystal $\mathrm{Sn}_{2} \mathrm{P}_{2} \mathrm{~S}_{6}$. It has been demonstrated as a promising material for fast hologram recording in the infrared $[16,17]$ and also interband photorefraction in the visible near $530 \mathrm{~nm}$ [18]. It has a transparency range extending from $0.53 \mu \mathrm{m}$ to $8 \mu \mathrm{m}$ and has been proposed as an interesting nonlinear optical material in this wide frequency range [19]. In $\mathrm{Sn}_{2} \mathrm{P}_{2} \mathrm{~S}_{6}$ crystal optical correlation at high repetition rates has been demonstrated [6] using interband recording at a wavelength of $532 \mathrm{~nm}$ using frequency doubled Nd:YAG laser. In the interband region the absorption constant has been previously calculated using the Urbach rule $[20,21]$.

The second material is near-stoichiometric $\mathrm{LiTaO}_{3}$ (SLT), which is attractive for several electro-optical, photorefractive or nonlinear optical applications like nonvolatile holographic data storage [22] or frequency doubling into the UV [23] and is transparent down to $\lambda \approx 280 \mathrm{~nm}$. In SLT deep UV fast interband photorefraction has recently been demonstrated [9]. The absorption constant in this regime has been measured with the method of the induced absorption [9] and by direct measurements in samples of different compositions [24].

\subsection{Determination of the absorption constant in $\mathrm{Sn}_{2} \mathrm{P}_{2} \mathrm{~S}_{6}$}

We first determined the absorption constants in $\mathrm{Sn}_{2} \mathrm{P}_{2} \mathrm{~S}_{6}$ using the direct transmission method. We used a thin plate of $\mathrm{Sn}_{2} \mathrm{P}_{2} \mathrm{~S}_{6}$ with dimensions $7.3 \times 8.3 \times 0.045 \mathrm{~mm}^{3}$ along the $x, y$ and $z$ axes. In this work we use the standard coordinate system with the $z$ axis parallel to the crystallographic $c$ axis, $y \| b$ normal to the mirror plane, and $x$ normal to $y$ and $z$. The thin plate was attached to a substrate of quartz with a thickness of $3 \mathrm{~mm}$. The crystal was poled along the $x$ axis. The absorption constant in the interband regime was measured by the direct transmission method at four Arion laser lines $\left(\lambda_{1}=514 \mathrm{~nm}, \lambda_{2}=501 \mathrm{~nm}, \lambda_{3}=496 \mathrm{~nm}\right.$, $\lambda_{4}=488 \mathrm{~nm}$ ). In all the experiments the contribution of the reflections between the substrate and the $\mathrm{Sn}_{2} \mathrm{P}_{2} \mathrm{~S}_{6}$ plate were taken into account as well as multiple Fresnel reflections in the crystal itself. We used the recently determined twooscillator Sellmeier parameters for the refractive indices of $\mathrm{Sn}_{2} \mathrm{P}_{2} \mathrm{~S}_{6}$ [25]. The results of the transmission measurement with the laser lines are given in Table 1 and Fig. 3. The solid lines in Fig. 3 are obtained with the Urbach extrapolation. This extrapolation uses the exponential behavior of the $a b$ sorption constant at the absorption edge:

$\alpha(v)=\alpha_{0} \exp \left(\frac{h v-E_{0}}{w}\right)$

where $w$ is the energy width of the exponential absorption edge, and $\alpha_{0}$ and $E_{0}$ empirical parameters. In the measurements reported in [21] it seems that the axes were defined differently, because the Urbach parameters given there would qualitatively match with our results if the polarizations $x$ and $y$ were exchanged. The difference in the absorption values obtained there might be related to a possible different crystal composition.

The $\mathrm{Sn}_{2} \mathrm{P}_{2} \mathrm{~S}_{6}$ crystal used for the photocurrent measurements had the dimensions $5.07 \times 5.38 \times 4.85 \mathrm{~mm}^{3}$ along the $x, y$ and $z$ axes. It was poled along the $x$ axis and had silver electrodes painted onto the $x$ surfaces. We applied an electric field between 200 and $600 \mathrm{~V} / \mathrm{cm}$ parallel to the $x$ axis, which is about 15 degrees off the spontaneous polarization [26]. The crystal surface normal to $z$ of the sample was homogeneously

\begin{tabular}{|c|c|c|c|c|}
\hline Wavelength & $\operatorname{direct} \alpha_{x}\left[\mathrm{~cm}^{-1}\right]$ & photoc. $\alpha_{x}\left[\mathrm{~cm}^{-1}\right]$ & direct $\alpha_{y}\left[\mathrm{~cm}^{-1}\right]$ & photoc. $\alpha_{y}\left[\mathrm{~cm}^{-1}\right]$ \\
\hline$\lambda_{1}=514 \mathrm{~nm}$ & $490 \pm 20$ & $380 \pm 120$ & $110 \pm 10$ & starting point \\
\hline$\lambda_{2}=501 \mathrm{~nm}$ & $1450 \pm 50$ & $1100 \pm 450$ & $500 \pm 20$ & $420 \pm 130$ \\
\hline$\lambda_{3}=496 \mathrm{~nm}$ & $1730 \pm 65$ & $1350 \pm 530$ & $860 \pm 35$ & $800 \pm 280$ \\
\hline$\lambda_{4}=488 \mathrm{~nm}$ & - & $2600 \pm 800$ & $1730 \pm 70$ & $2050 \pm 850$ \\
\hline
\end{tabular}
illuminated with green $\left(\lambda_{1}=514 \mathrm{~nm}, \lambda_{2}=501 \mathrm{~nm}\right)$ or blue $\left(\lambda_{3}=496 \mathrm{~nm}, \lambda_{4}=488 \mathrm{~nm}\right)$ light polarized either parallel to

TABLE 1 Absorption constants $\alpha_{x}$ and $\alpha_{y}$ of pure $\mathrm{Sn}_{2} \mathrm{P}_{2} \mathrm{~S}_{6}$ as obtained with a direct transmission measurement using an argon laser and a thin sample $(z=45 \mu \mathrm{m})$ and as obtained from photocurrent measurements with a bulk crystal 


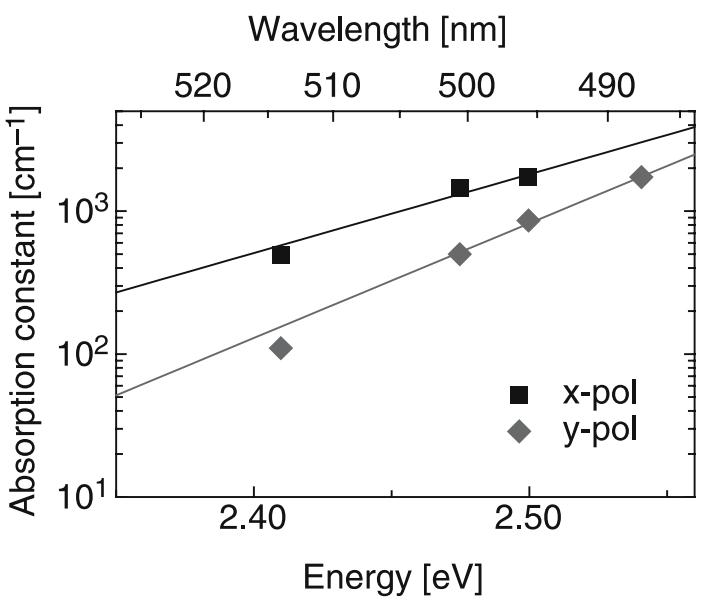

FIGURE 3 Absorption constant of $\mathrm{Sn}_{2} \mathrm{P}_{2} \mathrm{~S}_{6}$ in the interband regime measured with a thin sample of $45 \mu \mathrm{m}$ thickness. The solid lines are according to $(7)$

the $x$ or $y$ axis of the crystal. We determined the photocurrent by measuring the voltage drop over a reference resistor of $125 \mathrm{k} \Omega$ put in series with the crystal. A multimeter with a large input impedance ( $1 \mathrm{G} \Omega$ ) was used for the current measurement.

For increasing light intensity we expect the different ratios of $J$ to approach a constant value corresponding to (4)(6), because we approach the purely interband absorption regime. Figure 4 shows the intensity dependence of the ratio between the photocurrents $J_{x}^{\lambda_{4}} / J_{y}^{\lambda_{4}}$ of the $x$-polarization and the $y$-polarization at $\lambda_{4}=488 \mathrm{~nm}$. After reaching the intensity $10 \mathrm{~mW} / \mathrm{cm}^{2}$, the number of charge carriers in the band is sufficient for band to band recombination and the photocurrent follows the expected square root intensity dependence. In this regime the ratio $J_{x}^{\lambda_{4}} / J_{y}^{\lambda_{4}}$ is a constant and the ratio of the absorption constants $\alpha_{y}^{\lambda_{4}} / \alpha_{x}^{\lambda_{4}}$ can be determined. The results for $\lambda_{1}, \lambda_{2}, \lambda_{3}$ and $\lambda_{4}$ obtained from this and from the direct measurements with thin plates agree very well, as can be seen in Table 1 .

The absorption coefficient $\alpha_{x}^{\lambda_{4}}$ could not be measured directly by the classical method since the absorption constant was too high even for the thin plate of $45 \mu \mathrm{m}$ thickness. However with the photocurrent method, we determined $\alpha_{x}^{\lambda_{4}}=$ $2600 \pm 800 \mathrm{~cm}^{-1}$ which corresponds well with the Urbach ex-

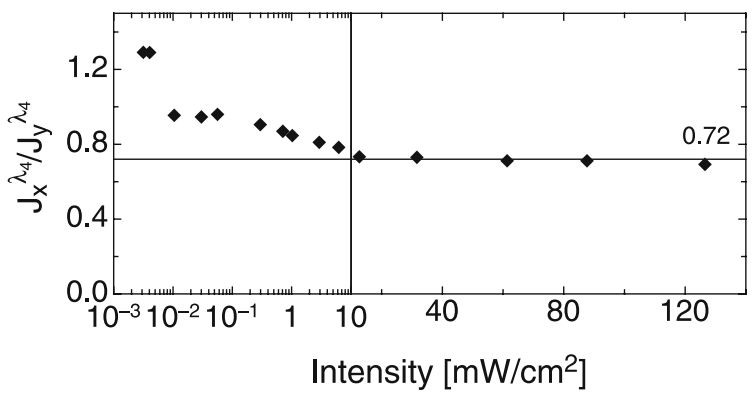

FIGURE 4 Ratio of the photocurrents $J_{x}^{\lambda_{4}} / J_{y}^{\lambda_{4}}$ of the $x$-polarization and the $y$-polarization as a function of the intensity for $\lambda_{4}=488 \mathrm{~nm}$ and $E=$ $600 \mathrm{~V} / \mathrm{cm}$. For intensities above $10 \mathrm{~W} / \mathrm{cm}^{2}$ a constant ratio $J_{x}^{\lambda_{4}} / J_{y}^{\lambda_{4}}=0.72$ is reached trapolation (see Fig. 3) of the directly measured absorption constants, which gave $\alpha_{x}^{\lambda_{4}}=3200 \mathrm{~cm}^{-1}$.

\subsection{Determination of the absorption constant in $\mathrm{LiTaO}_{3}$}

For the determination of the photocurrent in nearstoichiometric $\mathrm{LiTaO}_{3}$ (SLT) in the interband regime, we used UV light, provided by external cavity frequency doubling (WaveTrain, Spectra Physics) of the output of an Arion laser, producing continuous-wave laser radiation at $\lambda=$ $257 \mathrm{~nm}$. The near stoichiometric crystal with a composition $\mathrm{Li} /(\mathrm{Li}+\mathrm{Ta})=49.9 \%\left(\right.$ Curie temperature $\left.T_{\mathrm{C}}=684^{\circ} \mathrm{C}\right)$, was poled along the $z$ axis and had the dimensions $1.96 \times 7.94 \times$ $8.16 \mathrm{~mm}^{3}$ along the $x, y$ and $z$ axes which where chosen parallel to $a, b$ and $c$ crystal axes respectively. The absorption coefficients at $\lambda=257 \mathrm{~nm}$ were calculated using the Urbach extrapolation for the stoichiometry of our sample, using the data reported in [24], which gives $\alpha_{z}=270 \mathrm{~cm}^{-1}$ and $\alpha_{y}=265 \mathrm{~cm}^{-1}$. This results in a ratio of $\alpha_{y} / \alpha_{z}=0.98$, which agrees well with the value $\alpha_{y} / \alpha_{z}=0.92 \pm 0.10$ that we get from the photocurrent measurements. These results are also expected from direct measurements using a spectrophotometer, which show a similar absorption constant for both polarizations close to the absorption edge.

With a thin plate of magnesium doped near-stoichiometric $\mathrm{LiTaO}_{3}\left(\mathrm{Mg}: \mathrm{SLT}, T_{\mathrm{C}}=694^{\circ} \mathrm{C}\right)$ with dimensions $0.107 \times$ $9.8 \times 10.0 \mathrm{~mm}^{3}$ along the $x, y$ and $z$ axes we measured the absorption directly at $\lambda=257 \mathrm{~nm}$. Both polarizations showed almost the same absorption constants: $\alpha_{y}=700 \pm 40 \mathrm{~cm}^{-1}$ and $\alpha_{z}=690 \pm 40 \mathrm{~cm}^{-1}$. This confirms the data obtained with the new method, since the ratio of the two polarizations is supposed to be similar for this composition, although the absolute values differ [9, 24].

We previously used another method, the method of the induced absorption change to determine the absorption constant in our SLT crystal at $\lambda=257 \mathrm{~nm}$ [9]. These results however $\left(\alpha_{z}=450 \pm 70 \mathrm{~cm}^{-1}\right.$ and $\left.\alpha_{y}=170 \pm 30 \mathrm{~cm}^{-1}\right)$ do not correspond with the results presented here and determined by the photocurrent method and the results presented in [24]. In $\mathrm{BaTiO}_{3}$, however, the method of induced absorption allowed for an accurate determination of absorption constants up to $1400 \mathrm{~cm}^{-1}$ as has been shown in [8]. The failure of the induced absorption method in $\mathrm{LiTaO}_{3}$ samples may suggest that the photo-induced absorption is not, as required, a monotonic function of the light intensity over the whole intensity range. Our new simple photoconductivity-based method can therefore be used to independently check the results obtained by other more complex techniques.

\section{4}

\section{Conclusions}

We determined the absorption constants beyond the lower edge of the transmission region (absorption constant $10-10^{4} \mathrm{~cm}^{-1}$ ) with a good accuracy. This method does not require the knowledge of any other material constants and it does not require the preparation of thin samples or plates with polished sharp edges.

For $\mathrm{Sn}_{2} \mathrm{P}_{2} \mathrm{~S}_{6}$ the absorption constant has been determined from $488-514 \mathrm{~nm}$. The photocurrent measurements have 
been compared with the data obtained with the direct transmission measurement of the absorption in a very thin plate. These values match quite well and confirm the reliability of the new technique. Also for near-stoichiometric $\mathrm{LiTaO}_{3}$ the obtained data match the existing values and shows the advantage compared for instance to the indirect method based on the photo-induced absorption.

The technique presented here can be used for determining the absorption constants of any photoconductive crystal in which interband transitions are induced within a regime where the photoconductivity depends on the square root of the light intensity. Furthermore, provided that all the absorbed light gives rise to photoconduction, the same method can be adapted also to materials where the intensity dependence of the photoconductivity exhibits a regime with a characteristic exponent other than $1 / 2$ or 1 over a sufficiently broad range of light intensities.

ACKNOWLEDGEMENTS We thank J. Hajfler for his expert crystal preparation, Dr. A. Grabar for supplying the $\mathrm{Sn}_{2} \mathrm{P}_{2} \mathrm{~S}_{6}$ crystals and Dr. K. Kitamura for supplying the $\mathrm{LiTaO}_{3}$ crystals. This research has been supported by the Swiss National Science Foundation (NF 2-777416-04).

\section{REFERENCES}

1 N.M. Jokerst, E. Garmire, Appl. Phys. Lett. 53, 897 (1988)

2 D.D. Nolte, Q. Wang, M.R. Melloch, Appl. Phys. Lett. 58, 2067 (1991)

3 A. Partovi, A.M. Glass, D.H. Olson, G.J. Zydzik, K.T. Short, R.D. Feldmann, R.F. Austin, Opt. Lett. 17, 655 (1992)

4 P. Bernasconi, G. Montemezzani, M. Wintermantel, L. Biaggio, P. Günter, Opt. Lett. 24, 199 (1999)

5 P. Dittrich, G. Montemezzani, P. Bernasconi, P. Günter, Opt. Lett. 24, 1508 (1999)
6 R. Ryf, G. Montemezzani, P. Günter, A.A. Grabar, I.M. Stoika, Y.M. Vysochanskii, Opt. Lett. 26, 1666 (2001)

7 P. Dittrich, G. Montemezzani, P. Günter, Opt. Commun. 214, 363 (2002)

8 G. Ross, G. Montemezzani, P. Bernasconi, M. Zgonik, P. Günter, J. Appl. Phys. 79, 3665 (1996)

9 P. Dittrich, B. Koziarska-Glinka, G. Montemezzani, P. Günter, S. Takekawa, K. Kitamura, Y. Furukawa, J. Opt. Soc. Am. B 21, 632 (2004)

10 G. Montemezzani, P. Rogin, M. Zgonik, P. Günter, Phys. Rev. B 49, 2484 (1994)

11 M. Jazbinsek, M. Zgonik, S. Takekawa, M. Nakamura, K. Kitamura, H. Hatano, Appl. Phys. B 75, 891 (2002)

12 J.A. Bardwell, M.J. Dignam, J. Chem. Phys. 83, 5468 (1985)

13 L. Mutter, M. Jazbinsek, M. Zgonik, U. Meier, C. Bosshard, P. Günter, J. Appl. Phys. 94, 1356 (2003)

14 T.S. Moss, Rep. Prog. Phys. 28, 16 (1965)

15 K. Buse, Appl. Phys. B 64, 273 (1997)

16 S.G. Odoulov, A.N. Shumelyuk, U. Hellwig, R.A. Rupp, A.A. Grabar, Opt. Lett. 21, 752 (1996)

17 M. Jazbinsek, G. Montemezzani, P. Günter, A.A. Grabar, I.M. Stoika, Y.M. Vysochanskii, J. Opt. Soc. Am. B 20, 1241 (2003)

18 G. Montemezzani, R. Ryf, D. Haertle, P. Günter, A.A. Grabar, I.M. Stoika, Y.M. Vysochanskii, Ukr. J. Phys. 49, 333 (2004)

19 D. Haertle, M. Jazbinsek, G. Montemezzani, P. Günter, Opt. Express 13, $3765(2005)$

20 R. Ryf, G. Montemezzani, P. Günter, A.A. Grabar, I.M. Stoika, OSA Trends in Optics and Photonics 27, 80 (1999)

21 I.P. Studenyak, V.V. Mitrovcij, G.S. Kovacs, O.A. Mykajlo, M.I. Gurzan, Y.M. Vysochanskii, Ferroelectrics 254, 295 (2001)

22 Y.W. Liu, K. Kitamura, S. Takekawa, M. Nakamura, Y. Furukawa, H. Hatano, J. Appl. Phys. 95, 7637 (2004)

23 J.P. Meyn, M.M. Fejer, Opt. Lett. 22, 1214 (1997)

24 C. Baumer, C. David, A. Tunyagi, K. Betzler, H. Hesse, E. Kratzig, M. Wohlecke, J. Appl. Phys. 93, 3102 (2003)

25 D. Haertle, A. Guarino, J. Hajfler, G. Montemezzani, P. Günter, Opt. Express 13, 2047 (2005)

26 M.M. Maior, Y.M. Vysochanskii, I.P. Prits, B. Molnar, L.A. Seikovskaya, V.Y. Slivka, Sov. Phys. Cryst. 35, 767 (1990) 